\title{
Semirelativistic Bound States: (Pseudo-) Spinless-Salpeter Approaches Reassessed
}

\section{Wolfgang Lucha*}

Institute for High Energy Physics, Austrian Academy of Sciences, Nikolsdorfergasse 18, A-1050 Vienna, Austria

E-mail: Wolfgang. Lucha@oeaw.ac.at

Relativistic quantum field theory offers, in form of the homogeneous Bethe-Salpeter framework, a (Poincaré-covariant) description of bound states in terms of their underlying theory's fundamental degrees of freedom. In view of the intrinsic complexity of this approach, simplifications have been sought and abundantly found. The significance of these latter approximations may be estimated by comparing their predictions with (easily inferable) rigorous constraints on the bound-state spectra, such as existence, number and location of discrete eigenstates. The application of these techniques to selected proposed bound-state equations is exemplified for a large class of generalizations of the Hellmann potential frequently employed in several areas of science such as physics and chemistry.

European Physical Society Conference on High Energy Physics - EPS-HEP2019

10-17 July, 2019

Ghent, Belgium

\footnotetext{
* Speaker.
} 


\section{Different Semirelativistic Bound-State Treatments of Poincaré-Covariant Descent}

Firmly grounded within the framework of relativistic quantum field theories, the homogeneous Bethe-Salpeter equation [1] forms a Poincaré-covariant, albeit not always easy-to-handle, approach to bound states. Driven by the desire to obtain analytic, thus easier to control, yet still to some extent (semi-) relativistic bound-state treatments, more or less severe simplifications of the Bethe-Salpeter formalism have been proposed: Ignoring entirely all dependence on timelike variables generates the rather broad class of (merely) instantaneous Bethe-Salpeter equations [2]. Assuming, furthermore, also free propagation of the bound-state constituents leads to the Salpeter equation [3]. Skipping all negative-energy contributions and all reference to any spin degrees of freedom eventually yields the spinless Salpeter equation, the eigenvalue equation of a Hamiltonian $H$ generically consisting of the bound-state constituents' relativistic kinetic energy and a potential $V$ encoding all their interactions. For the case of bound states of two particles of equal masses $m$, each such Hamiltonian $H$ thus reads

$$
H \equiv 2 \sqrt{\boldsymbol{p}^{2}+m^{2}}+V(\boldsymbol{x}) .
$$

The nonlocality of this operator $H$ renders hard to find exact analytic solutions to its eigenvalue problem. In view of this, (rather bizarre) approximations to the spinless Salpeter equation have been proposed, by manipulations such as expanding kinetic energies to one order beyond the Schrödinger limit, arriving at operators not bounded from below, and inserting the Schrödinger limit into the thus fabricated pseudo-spinless-Salpeter equations, entirely ignoring the operator nature of the problem. Unsurprisingly, most of these pseudo-spinless-Salpeter attempts do not withstand rigorous scrutiny.

Definitely more reliable strategies rely on the derivation of rigorous statements on the spectrum of the operator $H$, such as establishing its boundedness from below (Sect. 3) or providing bounds on number (Sect. 5) and location (Sect. 4) of its discrete eigenvalues or validating approximate findings by the proper relativistic virial theorem $[4,5]$. The latter bulk of tools has been applied to relativistic problems defined by, for instance, interaction potentials $V(\boldsymbol{x})=V(r)$, with $r \equiv|\boldsymbol{x}|$, of Woods-Saxon [6,7], Hulthén [7,8], Yukawa [9], kink-like [10], and generalized-Hellmann [11] form. We highlight the capability of this programme for the illustrative set of generalized Hellmann potentials (Sect. 2).

Table 1: Classification of all generalized Hellmann potentials $V_{\mathrm{H}}(r)$ with respect to the size of the coupling $v$ of their Yukawa contributions relative to the nonvanishing coupling $\kappa \supsetneqq 0$ of their Coulomb contribution [11].

\begin{tabular}{|c|c|c|c|c|}
\hline $\begin{array}{l}\text { Boundedness } \\
\text { from below }\end{array}$ & $\begin{array}{l}\text { Characteristic } \\
\text { of potential }\end{array}$ & $\begin{array}{l}\text { Behaviour near } \\
\text { the origin } r=0\end{array}$ & $\begin{array}{l}\text { Sign of sum } \\
\text { of couplings }\end{array}$ & $\begin{array}{l}\text { Relation between } \\
\text { couplings } v \text { and } \kappa\end{array}$ \\
\hline unbounded & "singular" & $V_{\mathrm{H}}(r) \underset{r \rightarrow 0}{\longrightarrow}-\infty$ & $\kappa+v>0$ & $\begin{array}{c}v>\kappa \\
v=\kappa \\
0<v<\kappa \\
v=0 \\
-\kappa<v<0\end{array}$ \\
\hline bounded & $\begin{array}{l}\text { finite at origin } \\
\text { repulsive core }\end{array}$ & $\begin{array}{l}V_{\mathrm{H}}(r) \underset{r \rightarrow 0}{\longrightarrow} v b \\
V_{\mathrm{H}}(r) \underset{r \rightarrow 0}{\longrightarrow}+\infty\end{array}$ & $\begin{array}{l}\kappa+v=0 \\
\kappa+v<0\end{array}$ & $\begin{array}{l}v=-\kappa \\
v<-\kappa\end{array}$ \\
\hline
\end{tabular}




\section{Set of Generalized Hellmann-type Potentials: Classification by Overall Behaviour}

Broadening Hellmann's idea $[12,13]$ of the potential experienced by valence electrons in atoms to involve attractive Coulomb and repulsive Yukawa portions, the set of all superpositions (Fig. 1) of a Coulomb contribution, with nonnegative coupling $\kappa$, and a Yukawa-like contribution with positive range parameter $b$ and coupling $v$ of either sign defines the class of generalized Hellmann potentials

$$
V_{\mathrm{H}}(r) \equiv V_{\mathrm{C}}(r)+V_{\mathrm{Y}}(r)=-\frac{\kappa}{r}-v \frac{\exp (-b r)}{r}, \quad \kappa \geq 0, \quad v \gtreqless 0, \quad b>0 .
$$

Both individual components, tantamount to its parametric limits $v \rightarrow 0$, the Coulomb potential, and $\kappa \rightarrow 0$, the Yukawa potential, have been discussed thoroughly in Refs. [14-18] and [9], respectively.

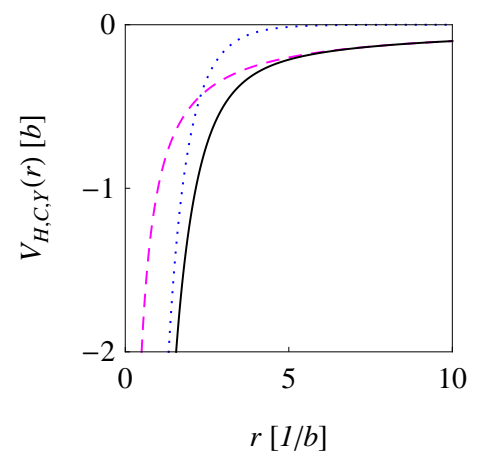

(a)

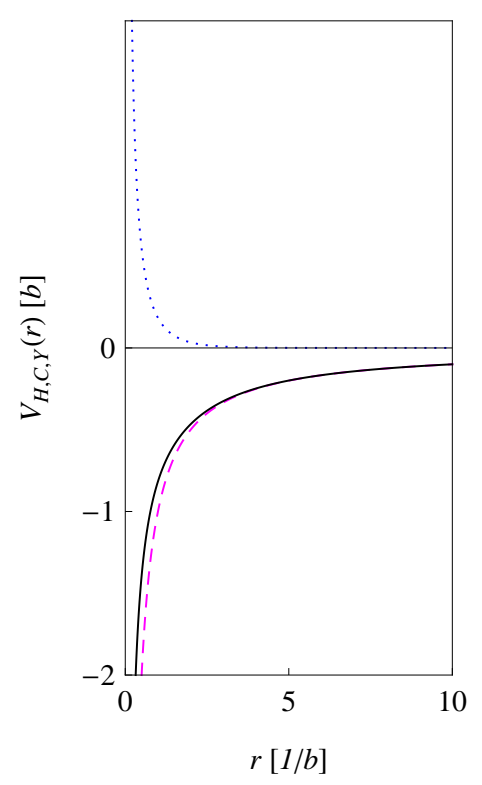

(d)

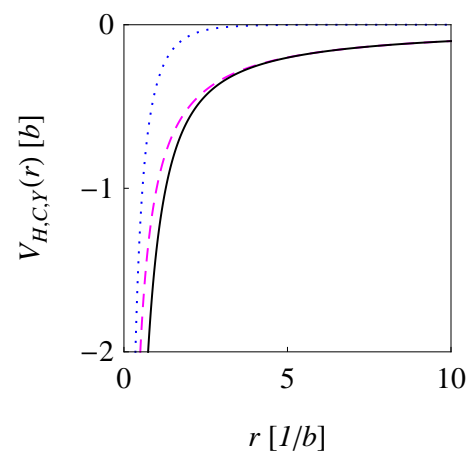

(b)

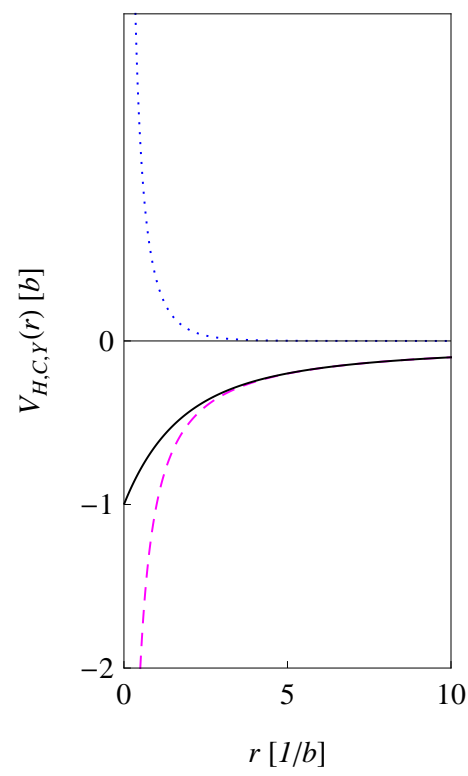

(e)

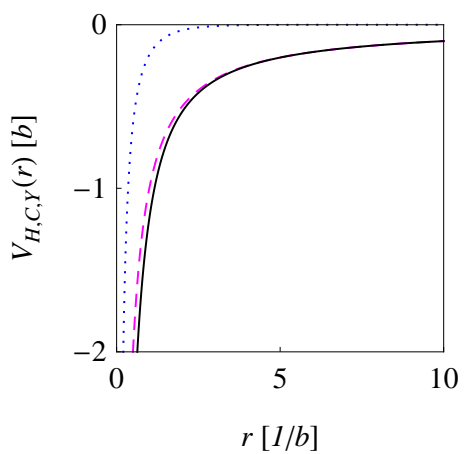

(c)

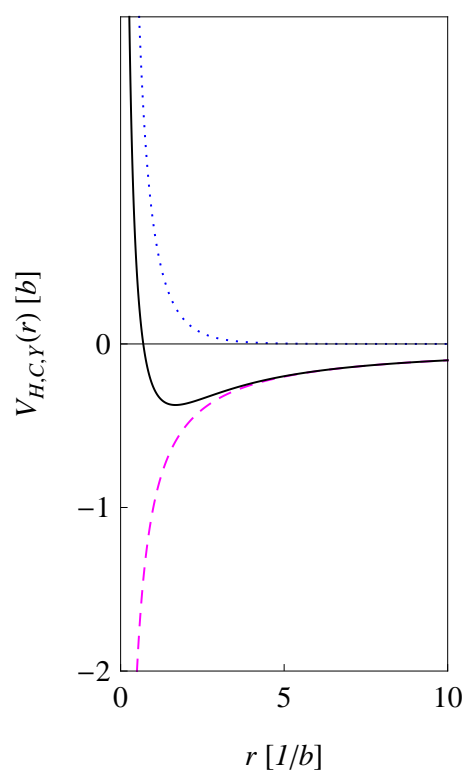

(f)

Figure 1: Samples of the six non-Coulombic types of (solid black) generalized Hellmann potentials $V_{\mathrm{H}}(r)$ of Table 1 [11], consisting of a (dashed magenta) Coulomb part $V_{\mathrm{C}}(r)$, with coupling $\kappa=1$, plus a (dotted blue) Yukawa part $V_{\mathrm{Y}}(r)$, with coupling $v=10(\mathrm{a}), v=1(\mathrm{~b}), v=0.5(\mathrm{c}), v=-0.5(\mathrm{~d}), v=-1$ (e), or $v=-2$ (f).

The types of arising spectra allow to subdivide this set into the seven categories of Table 1 [11]. 


\section{Eigenvalue Spectra and Coupling Parameters: Straightforward Rigorous Bounds}

The first goal in one's quest for bound states must be to identify constraints on the potential that ensure the semiboundedness of the spectrum $\sigma_{H}$ of the resulting spinless relativistic Hamiltonian $H$. For large distances $r$, every potential of generalized Hellmann shape approaches its Coulombic part:

$$
V_{\mathrm{H}}(r) \underset{r \rightarrow \infty}{\longrightarrow} V_{\mathrm{C}}(r) \text {. }
$$

This observation, in turn, entails that all discrete eigenvalues $E_{k}$ of $H$, related to the bound states, are bounded from above by $E_{k} \leq 0, k=0,1,2, \ldots$, in other words, all of these are definitely nonpositive.

"Nonsingular" Hellmann potentials are bounded from below [since they all satisfy $V_{\mathrm{H}}(r)>-\infty$ ] and thus, due to the nonnegativity $\sqrt{\boldsymbol{p}^{2}+m^{2}} \geq 0$ of any kinetic term, also their Hamiltonians:

$$
\begin{array}{lll}
H \geq V_{\mathrm{H}}(r) \geq \min _{0 \leq r<\infty} V_{\mathrm{H}}(r)>-\infty & \text { for } & v<-\kappa, \\
H \geq V_{\mathrm{H}}(r) \geq V_{\mathrm{H}}(0)=v b & \text { for } & v=-\kappa .
\end{array}
$$

"Singular" Hellmann potentials develop, because of the relation $v>-\kappa$ among their couplings, negative singularities at $r=0$ bounded from below by an associated Coulomb-like behaviour:

$$
V_{\mathrm{H}}(r) \geq-\frac{\alpha}{r} \quad \text { with }\left\{\begin{array}{llll}
\alpha=\kappa+v & \text { for } \quad v>0 & \Longleftrightarrow & V_{\mathrm{Y}}(r)<0, \\
\alpha=\kappa & \text { for } \quad v \leq 0 & \Longleftrightarrow & V_{\mathrm{Y}}(r) \geq 0 .
\end{array}\right.
$$

The spectra of the thereby defined relativistic Coulomb problems, $\sigma_{H}$, satisfy $[14,16]$, in turn,

$$
\sigma_{H} \geq 2 m \times \begin{cases}\sqrt{1-\left(\frac{\pi \alpha}{4}\right)^{2}} & \text { if and only if } \quad \alpha \leq \frac{4}{\pi}=1.273239 \ldots, \\ \sqrt{\frac{1+\sqrt{1-\alpha^{2}}}{2}} & \text { for } \quad \alpha \leq 1,\end{cases}
$$

proving their Hellmann counterparts to be, too, bounded from below. Exploiting the trial state

$$
\phi(\boldsymbol{x}) \propto \exp (-\mu r) \quad \Longleftrightarrow \quad \widetilde{\phi}(\boldsymbol{p}) \propto \frac{1}{\left(\boldsymbol{p}^{2}+\mu^{2}\right)^{2}}
$$

with $\mu>0$ in the expectation values of both kinetic term $\sqrt{p^{2}+m^{2}}[8,9]$ and potential $V_{\mathrm{H}}(r)$, it is trivial to establish that the couplings entering any Hellmann potential have to be bounded:

$$
\begin{aligned}
& \langle H\rangle=\left(\frac{16}{3 \pi}-\kappa-v\right) \mu+O(1) \underset{\mu \rightarrow \infty}{\longrightarrow}-\infty \quad \text { for } \quad \kappa+v>\frac{16}{3 \pi} \\
& \Longrightarrow\left\{\begin{aligned}
\kappa+v \stackrel{!}{\leq} \frac{16}{3 \pi}=1.69765 \ldots & \text { for } & v>0, \\
\kappa \stackrel{!}{\leq} \frac{16}{3 \pi}+|v| & \text { for } & -\kappa<v<0 .
\end{aligned}\right.
\end{aligned}
$$

For all those interested in a few more details, it might be advisable to consult, for instance, Ref. [19].

\section{Playing the Mini-Max Game: Variational Upper Bounds on Discrete Eigenvalues}

As soon as the boundedness from below of a reasonably defined Hamiltonian operator has been established, pinning down its bound states does make sense. On the basis of a characterization of its 
discrete spectrum by the famous minimum-maximum theorem, rigorous upper bounds on as well as improvable approximate estimates of the localization of bound-state energy levels can be derived by straightforward application of adequate variational techniques: a rather convenient trial-space basis utilizes products of generalized Laguerre orthogonal polynomials and spherical harmonics [20-22]. Table 2 shows bounds on energy levels for three of the six Yukawa-part-affected categories in Fig. 1.

Table 2: Bounds on the binding energies of low-lying eigenstates (identified by radial, $n_{r}$, and orbital angular momentum, $\ell$, quantum number) of the Hamiltonian (1.1) with generalized Hellmann potential (2.1) for three illustrative choices of the couplings $\kappa$ and $v$, and one common Yukawa range $b=m$. The upper bounds result from our trial-space basis, for parameters $\mu=m$ and $\beta=1$, the lower ones from Eqs. (3.1) through (3.3) [11].

\begin{tabular}{cc|ccc}
\hline & & \multicolumn{3}{c}{ Upper bound on $B_{k} \equiv E_{k}-2 m[m]\left(k \in \mathbb{N}_{0}\right)$} \\
\cline { 3 - 5 }$n_{r}$ & Bound state & $\kappa=v=\frac{1}{2}$ & $\kappa=1, v=-1$ & $\kappa=1, v=-2$ \\
\hline 0 & $\ell$ & {$[$ Fig. 1(b)] } & [Fig. 1(e)] & [Fig. 1(f)] \\
0 & 0 & -0.11673 & -0.17951 & -0.14410 \\
0 & 1 & -0.01579 & -0.06294 & -0.06157 \\
0 & 2 & -0.00616 & -0.02813 & -0.02812 \\
1 & 3 & - & -0.01553 & -0.01553 \\
1 & 0 & -0.02107 & -0.05464 & -0.04786 \\
1 & 1 & -0.00509 & -0.02810 & -0.02762 \\
1 & 2 & - & -0.01482 & -0.01481 \\
2 & 3 & - & -0.00624 & -0.00624 \\
2 & 0 & -0.00688 & -0.02566 & -0.02338 \\
2 & 1 & - & -0.01391 & -0.01356 \\
3 & 2 & - & -0.00122 & -0.00120 \\
\hline Lower bound on $B_{k}[m]$ & $-0.58578 \ldots$ & -1 & -0.00840 \\
\hline
\end{tabular}

The quality of the corresponding approximate variational eigenstates may be estimated by their fulfilment of the adequate, i.e., relativistic virial theorem [4,5] pertaining to any exact eigenstate $|\chi\rangle$ of any operator $H$ (defined, of course, by $H|\chi\rangle=E|\chi\rangle$ ), easily deducible for our Hamiltonian (1.1). (For those interested in slightly more details, it may be advisable to consult, for example, Ref. [19].)

\section{Discrete Spinless-Salpeter Energy Levels: Constraining Their Maximum Number}

A central issue in any spinless-Salpeter business is the actual number of bound states supported by a potential: how many bound states can one expect to find? In particular, one would like to know, at least: is their number finite or infinite? Unfortunately, in that context exact results are not abound.

A strict bound [23] on the number of spinless-Salpeter bound states exists for every nonpositive potential $V(\boldsymbol{x}) \leq 0$ that satisfies the merely finiteness-assuring constraint $V(\boldsymbol{x}) \in L^{3 / 2}\left(\mathbb{R}^{3}\right) \cap L^{3}\left(\mathbb{R}^{3}\right)$. For a rather simple reason, however, none of the generalized Hellmann potentials may belong to this set: for large $r$, due to the rapid decay of its Yukawa part any such potential approaches a Coulombic behaviour; the corresponding number of discrete energy eigenvalues thus will grow beyond bounds. Even if so, a spectral comparison theorem, recalled in Refs. [18,20-22,24], entails upper bounds on individual spinless-Salpeter energy levels, given by their nonrelativistic (Schrödinger) counterparts. 


\section{References}

[1] E. E. Salpeter and H. A. Bethe, Phys. Rev. 84 (1951) 1232.

[2] W. Lucha and F. F. Schöberl, J. Phys. G: Nucl. Part. Phys. 31 (2005) 1133, arXiv:hep-th/0507281.

[3] E. E. Salpeter, Phys. Rev. 87 (1952) 328.

[4] W. Lucha and F. F. Schöberl, Phys. Rev. Lett. 64 (1990) 2733.

[5] W. Lucha, Mod. Phys. Lett. A 5 (1990) 2473.

[6] W. Lucha and F. F. Schöberl, Int. J. Mod. Phys. A 29 (2014) 1450057, arXiv:1401.5970 [hep-ph].

[7] W. Lucha and F. F. Schöberl, EPJ Web Conf. 80 (2014) 00049, arXiv:1407.4624 [hep-ph].

[8] W. Lucha and F. F. Schöberl, Int. J. Mod. Phys. A 29 (2014) 1450181, arXiv:1408.4957 [hep-ph].

[9] W. Lucha and F. F. Schöberl, Int. J. Mod. Phys. A 29 (2014) 1450195, arXiv:1410.5241 [hep-ph].

[10] W. Lucha and F. F. Schöberl, Int. J. Mod. Phys. A 30 (2015) 1550062, arXiv:1412.4950 [hep-ph].

[11] W. Lucha and F. F. Schöberl, Int. J. Mod. Phys. A 34 (2019) 1950028, arXiv:1812.10756 [hep-ph].

[12] H. Hellmann, J. Chem. Phys. 3 (1935) 61.

[13] H. Hellmann and W. Kassatotschkin, J. Chem. Phys. 4 (1936) 324.

[14] I. W. Herbst, Commun. Math. Phys. 53 (1977) 285.

[15] I. W. Herbst, Commun. Math. Phys. 55 (1977) 316 (addendum).

[16] A. Martin and S. M. Roy, Phys. Lett. B 233 (1989) 407.

[17] J. C. Raynal, S. M. Roy, V. Singh, A. Martin, and J. Stubbe, Phys. Lett. B 320 (1994) 105.

[18] W. Lucha and F. F. Schöberl, J. Math. Phys. 41 (2000) 1778, arXiv:hep-ph/9905556.

[19] W. Lucha, arXiv:1908.08801 [hep-ph].

[20] W. Lucha and F. F. Schöberl, Int. J. Mod. Phys. A 14 (1999) 2309, arXiv:hep-ph/9812368.

[21] W. Lucha and F. F. Schöberl, Fizika B 8 (1999) 193, arXiv:hep-ph/9812526.

[22] W. Lucha and F. F. Schöberl, Recent Res. Dev. Phys. 5 (2004) 1423, arXiv:hep-ph/0408184.

[23] I. Daubechies, Commun. Math. Phys. 90 (1983) 511.

[24] W. Lucha and F. F. Schöberl, Phys. Rev. A 54 (1996) 3790, arXiv:hep-ph/9603429. 